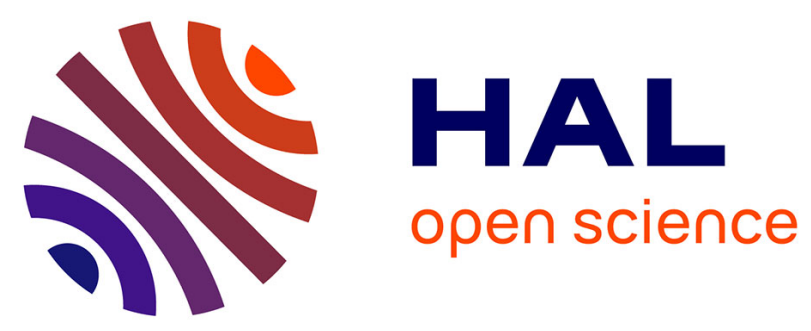

\title{
Stable Limit Cycle Generation for Underactuated Mechanical Systems, Application: Inertia Wheel Inverted Pendulum
}

\author{
Sébastien Andary, Ahmed Chemori, Sébastien Krut
}

\section{To cite this version:}

Sébastien Andary, Ahmed Chemori, Sébastien Krut. Stable Limit Cycle Generation for Underactuated Mechanical Systems, Application: Inertia Wheel Inverted Pendulum. IROS: Intelligent RObots and Systems, Sep 2008, Nice, France. pp.526-531, 10.1109/IROS.2008.4650994 lirmm-00305317

\section{HAL Id: lirmm-00305317 https://hal-lirmm.ccsd.cnrs.fr/lirmm-00305317}

Submitted on 23 Jul 2008

HAL is a multi-disciplinary open access archive for the deposit and dissemination of scientific research documents, whether they are published or not. The documents may come from teaching and research institutions in France or abroad, or from public or private research centers.
L'archive ouverte pluridisciplinaire HAL, est destinée au dépôt et à la diffusion de documents scientifiques de niveau recherche, publiés ou non, émanant des établissements d'enseignement et de recherche français ou étrangers, des laboratoires publics ou privés. 


\title{
Stable Limit Cycle Generation for Underactuated Mechanical Systems, Application: Inertia Wheel Inverted Pendulum
}

\author{
Sébastien Andary Ahmed Chemori Sébastien Krut \\ LIRMM, Univ. Montpellier 2 - CNRS, 161, rue Ada \\ 34392 Montpellier, France \\ andary (chemori, krut)@lirmm.fr
}

\begin{abstract}
This paper deals with a control approach dedicated to stable limit cycle generation for underactuated mechanical systems. The proposed approach is based on partial nonlinear feedback linearization and dynamic control for optimal periodic reference trajectories tracking. Simulation results and experiments show the efficiency of the proposed approach.
\end{abstract}

\section{INTRODUCTION}

Underactuated mechanical systems are those systems which have less control inputs than generalized coordinates (degrees of freedom) i.e they have generalized coordinates that are not actuated, and this is a source of dynamic contraints which are generally non integrable and therefore second order nonholonomic. Many examples of such systems exist, mainly in robotics, they include, among others, the underactuated robot manipulators, the gymnast robots and particularly the acrobot, the pendubot, the PVTOL (Planar Vertical Takeoff and Landing) aircrafts, the undersea vehicles and other mobile robots. An other basic feature of these class of systems is the nonlinear dynamics that they have, moreover their actuated coordinates are nonlinearly coupled with the unactuated coordinates. The underactuation in these mechanical systems is generally introduced by intentional design to reduce the manufacturing cost, the weight, and/or failure rate, so the obtained systems may be able to perform complex tasks with a reduced number of actuators, but they require new approaches to design effective control strategies, therefore they constitute a good framework of nonlinear control problems of both practical and theoretical interests, and for that they are attracting more and more attention of researchers from nonlinear control community.

In the literature many research efforts have been made on control aspects [2], [5], [8], [9], [4], [7], [1]. However, the field of control of such systems is still open to develop new control strategies.

Two stabilization control algorithms were presented in [2], namely a linear quadratic regulator based on partial feedback linearization and a sliding mode controller. Other partial feedback linearization based stabilization methods were presented in [5]. These controllers use iterative application of a finite time error contracting control law. In [8] partial feedback linearization is coupled with Lyapunov methods and energy shaping. Other control methods have been proposed such as passivity based control and bounded control [9], [6], [11]. In [1], a nonlinear predictive controller is proposed for both stabilization and stable limite cycle generation with an application to the ECP 505 inverted pendulum.

In this paper, a control approach based on collocated partial feedback linearization [9] and optimal reference trajectories is proposed, where the objective is the generation of stable limite cycles on both actuated and unactuated coordinates. The chosen application is the inertia wheel inverted pendulum. Experimental results are presented to show the efficiency of the proposed control scheme.

The paper is organized as follows. In section II the system used in our experiments is described as well as its dynamical model. The generation of the optimal reference trajectories as well as the control law are discussed in section III. In sections IV and V, simulation results as well as experiments are presented and discussed. Concluding remarks are drawn in section VI.

\section{The Inertia WheEl INVERTED PENDUlum}

The underactuated mechanical system studied in this paper is an inertia wheel inverted pendulum which consists of an inverted pendulum with a rotating wheel on it. Its mechanical structure is sketched in figure 1. The motor torque produces an angular acceleration of the rotating mass which generates a torque acting on the pendulum axis, therefore this last one can be controlled through the acceleration of the inertia wheel.

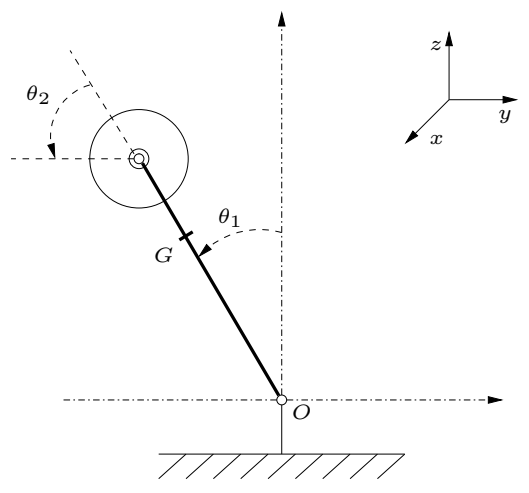

Fig. 1. the inertia wheel inverted pendulum 


\section{A. Dynamic modelling of the plant}

The dynamic model of the system is obtained by application of the Lagrange formulation [10], it is described by the following equations:

$$
\left[\begin{array}{cc}
i_{1}+i_{2} & i_{2} \\
i_{2} & i_{2}
\end{array}\right]\left[\begin{array}{l}
\ddot{\theta}_{1} \\
\ddot{\theta}_{2}
\end{array}\right]+\left[\begin{array}{c}
-\overline{m l} g \sin \left(\theta_{1}\right) \\
0
\end{array}\right]=\left[\begin{array}{l}
C_{1} \\
C_{2}
\end{array}\right]
$$

where $\theta_{1}$ and $\theta_{2}$ are respectively the angular position of the pendulum and the inertia wheel (cf. figure 1) and $i_{1}, i_{2}$ are respectively their moments of inertia. $C_{1}$ is the perturbation torque applied to the pendulum while $C_{2}$ is the torque developped by the motor. The perturbating torque $C_{1}$ is considered to be null in the design of the control approach. $\overline{m l}=m_{1} l_{1}+m_{2} l_{2}$ with $m_{1}$ and $m_{2}$ the mass of the pendulum and the inertia wheel and $l_{1}, l_{2}$ (respectively) are distances from origin $O$ to gravity center of the pendulum and the rotating mass respectively. In next section the proposed control scheme is detailed.

\section{The PROPOSED CONTROL SCHEME}

The objective of the control approach is to generate stable limite cycles on both actuated and unactuated coordinates of the system. This last one is underactuated since it has only one control input and two degrees of freedom. The control input will be used to track reference trajectories on only one coordinate. The question that may be asked is the following: which coordinate will be used to track the reference trajectories?

The application of collocated partial feedback linearization enables us to linearize completely the dynamics of the unactuated coordinate $\theta_{1}$, whereas the remaining nonlinear dynamics of $\theta_{2}$ constitute what we call the internal dynamics of the system. In order to ensure the stability of this internal dynamics, the reference trajectories have to be optimized. The optimization criterion is based on the rotation speed of the inertia wheel, that has to be minimized. Therefore, the idea is to propose parameterized reference trajectories, and compute their parameters by resolving the optimisation problem. In the following section the generation of the reference trajectories is discussed.

\section{A. Reference trajectory generation}

In order to generate reference trajectories, a derivable continuous periodical function needs to be defined. The reference trajectory is splitted up in half-period and each part is expressed by a parameterized polynomial function such that the reference trajectory can be written as:

$$
\theta_{1}^{r e f}(t)=f(t, p)=\left\{\begin{aligned}
A P_{p}\left(\frac{2 t}{\tau}\right) & \text { if } t \in\left[0, \frac{\tau}{2}[\right. \\
-A P_{1-p}\left(\frac{2 t}{\tau}-1\right) & \text { if } t \in\left[\frac{\tau}{2}, \tau[\right.
\end{aligned}\right.
$$

where $A$ and $\tau$ are respectively the amplitude and the period of the oscillation, $p$ is the optimization parameter. The polynomial $P_{p}(t)$ has to be normalized, it will vary from 1 at $t=0$ to -1 at $t=1$ for all $p$. In order to smoothly join successive parts of the reference trajectory, we constrain the first and second order derivatives of $P$ to be zero at $t=0$ and $t=1$. Finally, $p$ is such that $P_{p}(p)=0$. We can therefore define $P$ as a six-degree parameterized polynomial $P_{p}(t)$ :

$$
P_{p}(t)=\sum_{i=0}^{6} \alpha_{i}(p) t^{i}
$$

The consideration of the constraints leads to the following:

$$
\begin{array}{r}
P_{p}(0)=1 ; P_{p}(1)=-1 ; \frac{d P_{p}}{d t}(0)=0 ; \frac{d P_{p}}{d t}(1)=0 ; \\
\frac{d^{2} P_{p}}{d t^{2}}(0)=0 ; \frac{d^{2} P_{p}}{d t^{2}}(1)=0 ; P_{p}(p)=0
\end{array}
$$

Solving the above equations leads to the following:

$$
\begin{aligned}
& \alpha_{6}(p)=\frac{(2 p-1)\left(6 p^{4}-12 p^{3}+4 p^{2}+2 p+1\right)}{p^{3}(p-1)^{3}} \\
& \alpha_{5}(p)=\frac{-3\left(4 p^{6}-18 p^{4}+16 p^{3}-1\right)}{p^{3}(p-1)^{3}} \\
& \alpha_{4}(p)=\frac{3\left(10 p^{6}-18 p^{5}+10 p^{3}-1\right)}{p^{3}(p-1)^{3}} \\
& \alpha_{3}(p)=\frac{-\left(20 p^{6}-48 p^{5}+30 p^{4}-1\right)}{p^{3}(p-1)^{3}} \\
& \alpha_{2}(p)=0 \\
& \alpha_{1}(p)=0 \\
& \alpha_{0}(p)=1
\end{aligned}
$$

Figure 2 shows resulting trajectory for different values of $p$. Note that the pendulum spends as much time on

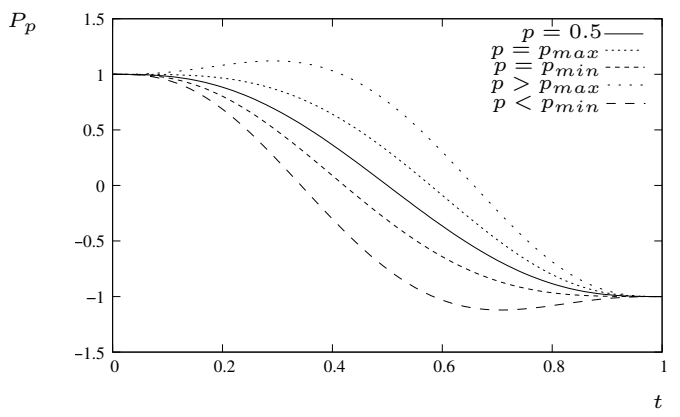

Fig. 2. normalized reference trajectory during half a period

both sides of the equilibrium point when $p=\frac{1}{2}$.

We want the pendulum passive joint (corresponding to $\left.\theta_{1}\right)$ to remain within interval $[-A, A]$, therefore only a subinterval $\left[p_{\min }, p_{\max }\right]$ of $[0,1]$, is admissible for $p$ values. This requirement can be written for a normalized trajectory as follows:

$$
\left.\forall p \in\left[p_{\min }, p_{\max }\right], \forall t \in\right] 0,1\left[,\left|P_{p}(t)\right|<1\right.
$$

$p_{\min }$ and $p_{\max }$ can be computed as follow. Assume that we have for some $p$ :

$$
\left.\exists t_{1} \in\right] 0,1\left[,\left|P_{p}\left(t_{1}\right)\right| \geq 1\right.
$$

Since $P_{p}(0)=1, P_{p}(1)=-1$ and $(5), P$ must admit at least one null tangency point. It means that at least one 
root of $\frac{d P_{p}}{d t}(t)$ is within ]0,1[. Let us compute the first derivative of $P_{p}(t)$ :

$$
\frac{d P_{p}}{d t}(t)=0 \Longrightarrow t=\frac{20 p^{6}-48 p^{5}+30 p^{4}-1}{24 p^{5}-60 p^{4}+40 p^{3}-2}
$$

The root expressed in (6) is the only real root satisfying the constraints discussed above. The two extremal trajectory $P_{p_{\text {min }}}(t)$ and $P_{p_{\text {max }}}(t)$ correspond respectively to values 1 and 0 for (6). This leads us to solve the following two equations:

$$
\begin{aligned}
& \frac{20 p^{6}-48 p^{5}+30 p^{4}-1}{24 p^{5}-60 p^{4}+40 p^{3}-2}=1 \\
& \frac{20 p^{6}-48 p^{5}+30 p^{4}-1}{24 p^{5}-60 p^{4}+40 p^{3}-2}=0
\end{aligned}
$$

Equation (7) admits only one real solution $p=p_{m i n}$, and (8) admits $p=p_{\max }$ as solution, both in interval $[0,1]$

\section{B. Tracking control law}

The equation (1) can be rewritten as follows:

$$
\begin{aligned}
& \ddot{\theta}_{1}=\frac{1}{i_{1}}\left(C_{1}-C_{2}+\overline{m l} g \sin \theta_{1}\right) \\
& \ddot{\theta}_{2}=\frac{-1}{i_{1}} C_{1}+\frac{\left(i_{1}+i_{2}\right)}{i_{1} i_{2}} C_{2}+\frac{-\overline{m l} g}{i_{1}} \sin \theta_{1}
\end{aligned}
$$

The above equations describe the system dynamics in open loop. According to the collocated partial feedback linearization, consider the following control law ${ }^{1}$ :

$$
\begin{aligned}
C_{2} & =\overline{m l} g \sin \left(\theta_{1}\right)-i_{1} \ddot{\theta}_{1}^{\text {ref }} \\
& +i_{1} k_{p}\left(\theta_{1}-\theta_{1}^{r e f}\right)+i_{1} k_{v}\left(\dot{\theta}_{1}-{\dot{\theta_{1}}}^{\text {ref }}\right)
\end{aligned}
$$

Where $k_{p}$ and $k_{v}$ are respectively the proportional and derivative gains and are used to tune the dynamics of the closed loop system. Injecting this control law in equation (9) leads to the following closed loop system:

$$
\ddot{e}+k_{v} \dot{e}+k_{p} e=0
$$

Where $e=\theta_{1}-\theta_{1}^{r e f}, \dot{e}=\dot{\theta}_{1}-\dot{\theta}_{1}^{r e f}$ and $\ddot{e}=\ddot{\theta}_{1}-\ddot{\theta}_{1}^{r e f}$. The resulting system is an asymptotically stable linear system with $k_{p}$ and $k_{v}$ positives gains [3]. Therefore $e, \dot{e} \rightarrow 0$ and thus $\theta_{1} \rightarrow \theta_{1}^{r e f}$ and $\dot{\theta}_{1} \rightarrow \dot{\theta}_{1}^{r e f}$. The pendulum joint then follows the reference trajectory $\theta_{1}^{r e f}(t)$

\section{Trajectory optimization}

The aim of trajectory optimization is to minimize the motor rotating speed as far as possible during tracking. That is, for a given initial value $\dot{\theta}_{2}(0)$, choosing the optimal value of $p$ minimizing $\dot{\theta}_{2}(\tau)$. From the set of state equations (9), (10) and from control law (11), the following set of differential equations describing the dynamics of the system in closed loop can be written as:

$$
\begin{aligned}
\ddot{\theta}_{1}= & \ddot{\theta}_{1}^{\text {ref }}-k_{v}\left(\dot{\theta}_{1}-\dot{\theta}_{1}^{\text {ref }}\right)-k_{p}\left(\theta_{1}-\theta_{1}^{\text {ref }}\right) \\
\ddot{\theta}_{2}= & -\frac{i_{1}+i_{2}}{i_{2}}\left(\ddot{\theta}_{1}^{r e f}-k_{v}\left(\dot{\theta}_{1}-\dot{\theta}_{1}^{r e f}\right)-k_{p}\left(\theta_{1}-\theta_{1}^{r e f}\right)\right)+ \\
& +\frac{\overline{m l} g}{i_{2}} \sin \left(\theta_{1}\right)
\end{aligned}
$$

\footnotetext{
${ }^{1}$ The torque $C_{1}$ is zero since the nominal system is not subject to external disturbing torque.
}

Let $g\left(p, \theta_{1}(0), \dot{\theta}_{1}(0), \dot{\theta}_{2}(0)\right)$ be a quadratic function of the angular velocity $\dot{\theta}_{2}$ at the end of a period during trajectory tracking with initial values $\theta_{1}(0), \dot{\theta}_{1}(0)$ and $\dot{\theta}_{2}(0)$. Remember from (2) that reference trajectory is parameterized by $p$. The optimization problem can then be formulated as:

$$
p^{o p t}=\arg \min _{p} g\left(p, \theta_{1}(0), \dot{\theta}_{1}(0), \dot{\theta}_{2}(0)\right)
$$

This problem is to be resolved at the beginning of each cycle, the solution enables us to define completely the reference trajectories to be tracked on the whole cycle.

\section{Simulation Results}

In this section simulation results are presented. They attest the feasibility of the proposed control scheme. Simulation results are obtained using Matlab software. Table I gives a description of control approach parameters. The

\begin{tabular}{|c|l|c|}
\hline Parameter & Signifiance & Value \\
\hline$A$ & Amplitude of the oscillation & $3 \mathrm{deg}$ \\
\hline$\tau$ & Period of the oscillation & $2 s$ \\
\hline$k_{p}$ & Proportionnal gain & 300 \\
\hline$k_{v}$ & Derivative gain & 20 \\
\hline$\theta_{1}(0)$ & Pendulum initial position & $10 \mathrm{deg}$ \\
\hline$\dot{\theta}_{1}(0)$ & Pendulum initial velocity & $0 \mathrm{deg} / s$ \\
\hline$\dot{\theta}_{2}(0)$ & Inertia wheel initial velocity & $0 \mathrm{deg} / s$ \\
\hline
\end{tabular}

TABLE I

PARAMETER DESCRIPTION AND VALUE

choice of initial value of $\theta_{1}$ is motivated by the physical properties of the inverted pendulum, as the pendulum angle value is $10 \mathrm{deg}$ at a standstill. Figure 3 displays overall simulation results. Figures $3(\mathrm{a})$ and $3(\mathrm{~b})$ show the pendulum joint position and velocity versus time in solid lines, while the dashed lines represent the reference position and velocity. These two figures show clearly the convergence of position and velocity to their reference trajectories. The inertia wheel velocity versus time is displayed in figure 3(c) where we observe that within two periods, the motor velocity reaches a limit cycle and oscillates around zero. Figure 3(d) represents control input which consists in the motor voltage; it remains in the admissible limits. The phase portrait $\left(\theta_{1}, \dot{\theta}_{1}\right)$ is depicted in figure 3(e) showing clearly the convergence from the initial condition to a stable limit cycle. Finally, figure 3(f) represents the absolute value of motor velocity versus absolute motor torque and the admissible region of the actuator given by the manufacturer of the DC motor where it can be seen that the trajectories remain inside the admissible region.

\section{ExPERIMENTAL RESUlts}

In this section experimental results are represented whith implementation issues, it starts with a description of inverted pendulum testbed then two experiments are represented. The first one concerns the control of the real system without perturbations; however in the second one the system is subject to an external perturbation. 


\section{A. Testbed and implementation issues}

Real time experiments are performed on an inertia wheel inverted pendulum developed at LIRMM (cf. figure 4). The pendulum angle $\theta_{1}$ is constrained to remain within the interval $\left[-10^{\circ}, 10^{\circ}\right]$ due to mechanical stops. The actuator of the system is a Maxon EC-powermax 30 DC motor and is equipped with an incremental encoder which allows the calculator to acquire the position of the inertia wheel joint in real time. In order to mesure the angle of the pendulum with respect to the vertical, the system is equipped with the inclinometer FAS-G of Micro-strain. The system is controlled with a computer equipped with $2.4 \mathrm{GHz}$ Intel processor. The control approach is implemented using $\mathrm{C}$ language, and the whole system is running under Ardence RTX real time OS based on a patched MS Windows kernel.

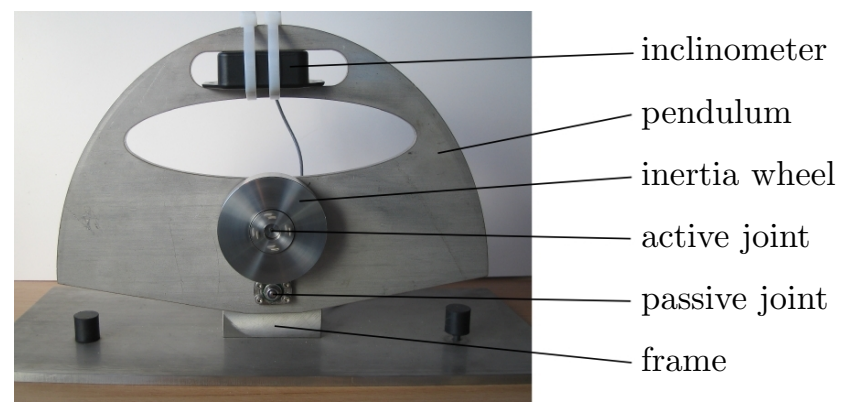

Fig. 4. The inertia wheel inverted pendulum

The proposed control scheme requires to resolve online an optimization problem, however due to computing time, real time implementation of such optimization is performed using a lookup table. Indeed a grid is defined for different initial values on $\dot{\theta}_{2}$, and the optimization problem (15) is resolved (off-line) for each value on the grid and stored in a table. Then the real time application of the proposed control scheme uses this lookup table to obtain instantaneously the optimization parameter.

\section{B. Real time experiments}

In this section real time experiments are presented and discussed. They are performed on the inverted pendulum testbed presented in the previous section. In the proposed experiment concerns the application of the proposed control approach to the system subject to external disturbance. Figure 5 shows overall results. In this experiment, perturbations are carried out by pushing the pendulum, which generates an external ponctual torque applied to the pendulum joint at approximately $t=10 \mathrm{~s}$ and $t=35 \mathrm{~s}$. Figure 5(a) represents pendulum joint position and its corresponding reference trajectory. In figure 5(b), where pendulum joint velocity and reference velocity are represented, perturbations can be observed as peaks. External perturbation compensation can be observed in figures 5(c) and 5(d) where, respectively, inertia wheel velocity and motor driver input voltage are displayed. We clearly observe peaks at the instant of disturbance and how the system brings back the motor velocity oscillation around zero after each one. The phase portrait and motor admissible power are displayed respectively in figures 5(e) and 5(f). The limit cycle is joined after each perturbation. Figure 5(g) represents the evolution of optimization parameter versus time (this parameter is normalized, thus it stays within the interval $[-1,1])$. The controller is able to keep the system around the reference trajectories and reject external disturbances introduced as unpredicted torques applied on the pendulum axis in a ponctual manner.

\section{Conclusion AND Future WORK}

In this paper a control approach is proposed for generation of stable limit cycles for underactuated mechanical systems. The control scheme is designed in the special case of the inertia wheel inverted pendulum, however it can be easily generalized to the case of underactuated mechanical systems. It is based on partial feedback linearization and optimal reference trajectories. The obtained results are presented as simulations and experiments on the inertia wheel inverted pendulum testbed. We are currently working on the optimization problem. The objective is to find an analytical solution of this last one for the linearized system. Furthermore, the compensation delay and the latency in response observed during real time experiments can be reduced by finding a more flexible reference trajectory, as well as choosing an optimal parameter at a higher frequency (currently once a period).

\section{REFERENCES}

[1] A. Chemori and M. Alamir. Nonlinear predictive control of under-actuated mechanical systems, application: the ECP 505 inverted pendulum. In MTNS'06 Proc. Mathematical Theory of Networks and Systems, 2004.

[2] V. Coverstone-Carroll K. Lee. Control algorithms for stabilizing underactuated robots. Journal of Robotic Systems, 15(12):681-697, 1998.

[3] H. Khalil. Nonlinear Systems. Prentice Hall, Upper Saddle River, Second Edition, 1996.

[4] K. Lee and V. Coverstone-Carroll. Control algorithms for stabilizing underactuated robots. Journal of Robotic Systems, 15(12):681-697, 1998.

[5] A. De Luca, R. Mattone, and G. Oriolo. Stabilization of an underactuated planar $2 \mathrm{r}$ manipulator. Int. J. Robust and Nonlinear Control, 10:181-198, 2000.

[6] T. McGeer. Passive dynamic walking. Int. J. of Robotics Research, 9(2):62-82, 1990.

[7] M. Spong. The swing up control problem for the acrobot. IEEE Control Systems Magazine, 15(1):49- 55, February 1995.

[8] M. Spong. Energy based control of a class of underactuated mechanical system. In Proc. 13th IFAC World Congress, volume $\mathrm{F}$, pages 431-436, 1996.

[9] M. Spong. Underactuated mechanical systems. Control Problems in Robotics and Automation, 230:135-150, 1998.

[10] M. Spong and M. Vidyasagar. Robot Dynamics and Control. John Wiley and Sons, 1989.

[11] A. Teel. Using saturation to stabilize a class of single-input partially linear composite systems. In Proc. NOLCOS, pages 24-28, 1992. 


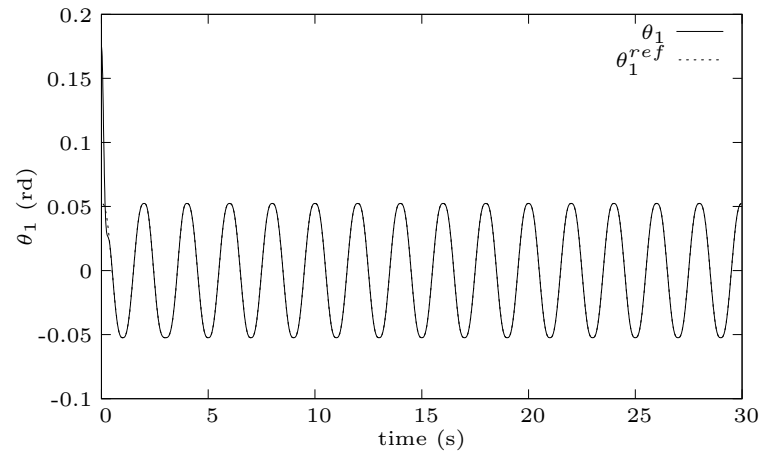

(a) pendulum axis position

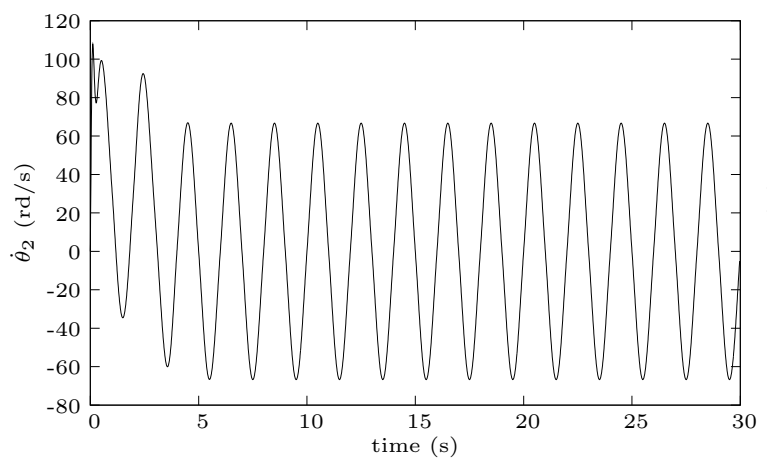

(c) inertia wheel rotation speed

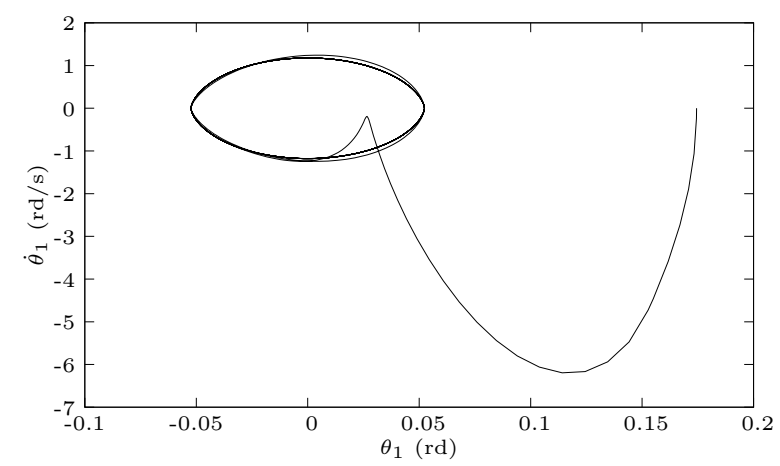

(e) phase portrait

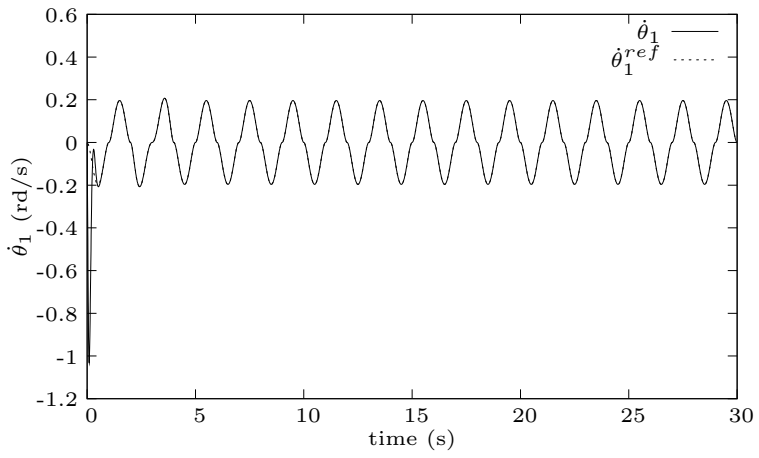

(b) pendulum axis velocity

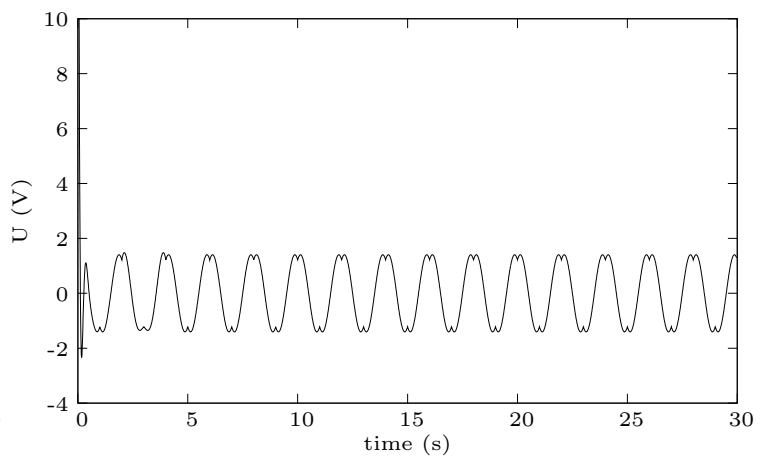

(d) motor driver input

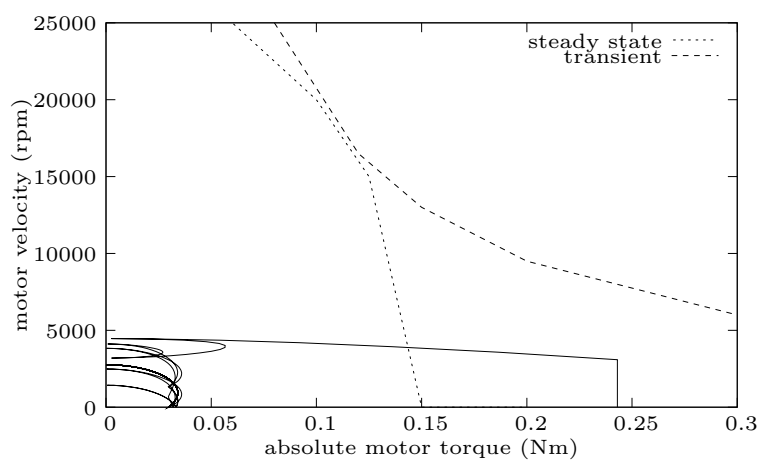

(f) admissible region of motor power

Fig. 3. Simulation results with $A=3 \mathrm{deg}, \tau=2 \mathrm{~s}$ 


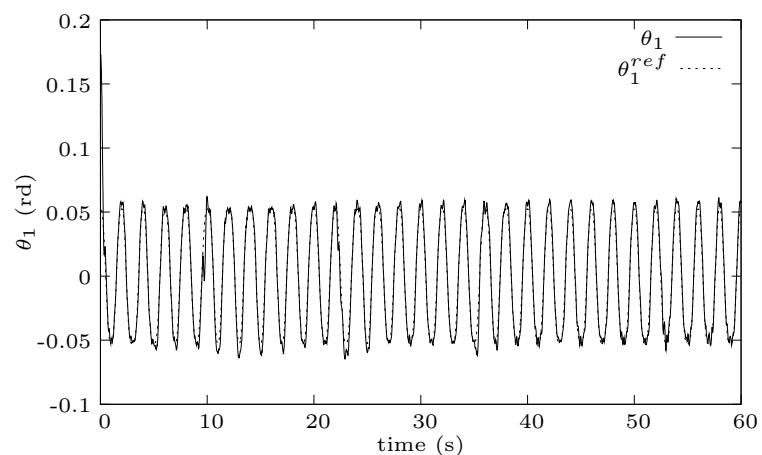

(a) pendulum axis position

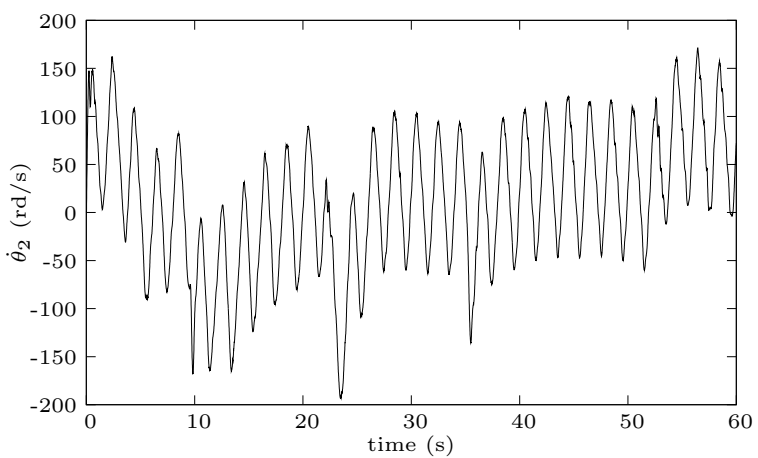

(c) inertia wheel rotation speed

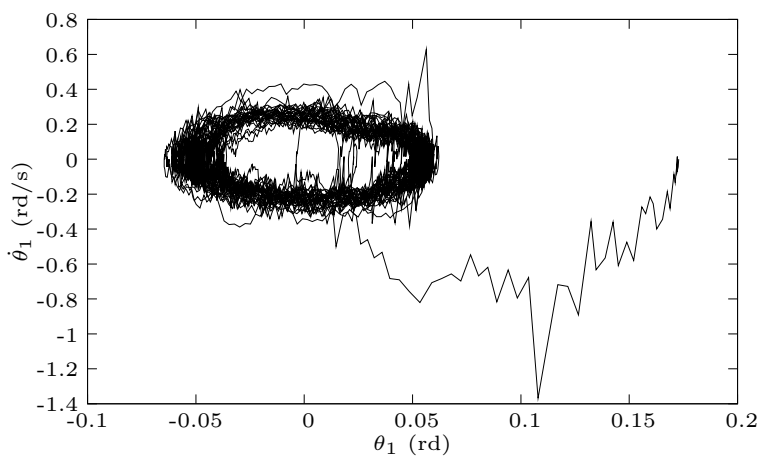

(e) phase portrait

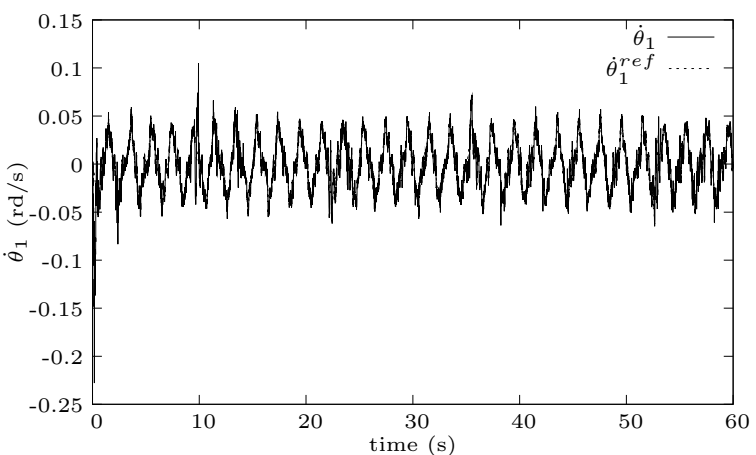

(b) pendulum axis velocity

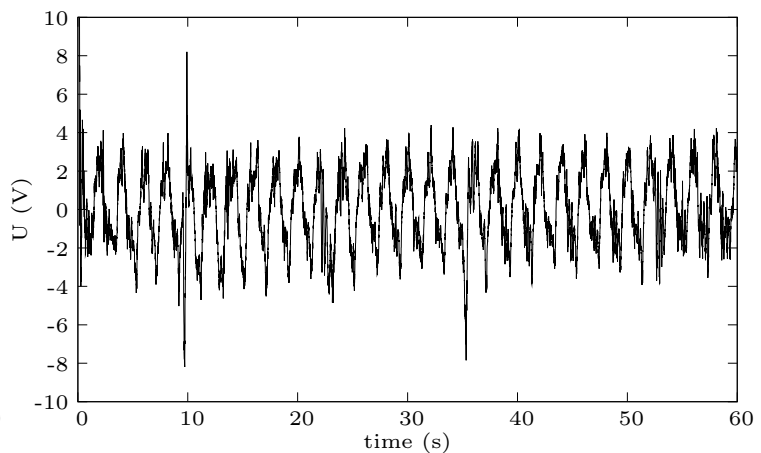

(d) motor driver input

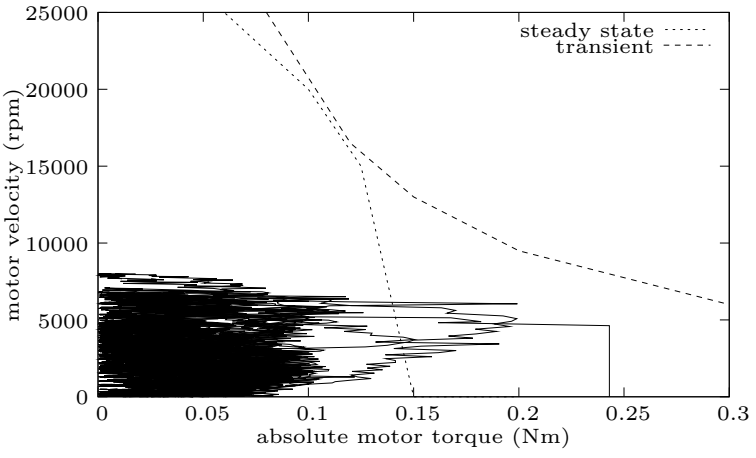

(f) admissible region of motor power

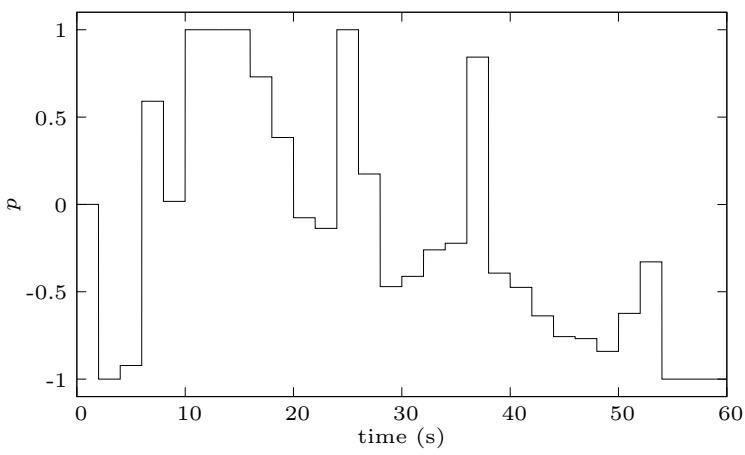

(g) evolution of the normalized optimization parameter

Fig. 5. Real time results with $A=3 \mathrm{deg}, \tau=2 \mathrm{~s}$, with external perturbations 